\title{
Joint segmentation of multivariate time series with hidden process regression for human activity recognition
}

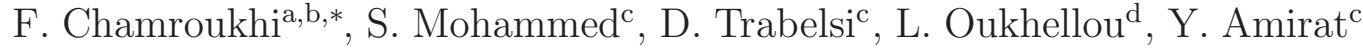 \\ ${ }^{a}$ Université de Toulon, CNRS, LSIS, UMR 7296, 83957 La Garde, France \\ ${ }^{b}$ Aix Marseille Université, CNRS, ENSAM, LSIS, UMR 7296, 13397 Marseille, France \\ ${ }^{c}$ University Paris-Est Créteil (UPEC), LISSI, 122 rue Paul Armangot, 94400, \\ Vitry-Sur-Seine, France \\ ${ }^{d}$ University Paris-Est, IFSTTAR, GRETTIA, F-93166 Noisy-le-Grand, France
}

\begin{abstract}
The problem of human activity recognition is central for understanding and predicting the human behavior, in particular in a prospective of assistive services to humans, such as health monitoring, well being, security, etc. There is therefore a growing need to build accurate models which can take into account the variability of the human activities over time (dynamic models) rather than static ones which can have some limitations in such a dynamic context. In this paper, the problem of activity recognition is analyzed through the segmentation of the multidimensional time series of the acceleration data measured in the 3 -d space using body-worn accelerometers. The proposed model for automatic temporal segmentation is a specific statistical latent process model which assumes that the observed acceleration sequence is governed by sequence of hidden (unobserved) activities. More specifically, the proposed approach is based on a specific multiple regression model incorporating a hidden discrete logistic process which governs the switching from one activity to another over time. The model is learned
\end{abstract}

\footnotetext{
*Corresponding author: Faicel Chamroukhi Université de Toulon, LSIS, UMR CNRS 7296

Bâtiment R, BP 20132 - 83957 La Garde Cedex, France

Tel: $+33(0) 494142006$

Fax: +33(0) 494142897

Email addresses: faicel.chamroukhi@univ-tln.fr (F. Chamroukhi ), samer.mohammed@u-pec.fr (S. Mohammed), dorra.trabelsi@u-pec.fr (D. Trabelsi), latifa.oukhellou@ifsttar.fr (L. Oukhellou), amirat@u-pec.fr (Y. Amirat)
} 
in an unsupervised context by maximizing the observed-data log-likelihood via a dedicated expectation-maximization (EM) algorithm. We applied it on a real-world automatic human activity recognition problem and its performance was assessed by performing comparisons with alternative approaches, including well-known supervised static classifiers and the standard hidden Markov model (HMM). The obtained results are very encouraging and show that the proposed approach is quite competitive even it works in an entirely unsupervised way and does not requires a feature extraction preprocessing step.

Keywords: Human activity recognition, inertial sensors, acceleration data, times series segmentation, hidden process regression, unsupervised learning, expectation-maximization algorithm

\section{Introduction}

The problem of human activity recognition is central for understanding and predicting the human behavior, in particular for a prospective of assistive services to elderly people. In fact, assistive services related to the aging population has gained an increasing attention in the last decades due the fact that this population is increasing and having more socio-economic impact. The aim is therefore to facilitate the daily lives of elderly or dependent people at home, increase their autonomy and improve their safety. The emergence of novel adapted technologies such as wearable and ubiquitous technologies is becoming a privileged solution to provide assistive services to humans, such as health monitoring, well being, security, etc. For example, the recognition of the activity from data measured by sensors can play important role in minimizing the risk of human fall (Hirata et al., 2008; Kangas et al., 2008; Noury et al., 2009; Lindemann et al., 2005). In addition, remote monitoring based systems can reduce considerably the amount of admission to hospitals by early detection of gradual deterioration in elderly health status (Scanaill et al., 2006).

In the context of activity recognition from measurements, the main measurement techniques used to quantify human activities are based on the use of inertial sensors (Altun et al., 2010; Trabelsi et al., 2011; Preece et al., 2009; Kavanagh and Menz, 2008; Parkka et al., 2006). Other sensors are also used to recognize human activities such as video-based systems (Cappozzo et al., 1996, 2005; Aminian et al., 1999), goniometers (Kostov et al., 1995), elec- 
tromyography (EMG) (Hussein and Granat, 2002), etc. However, the videobased approach is less adapted regarding particularly the personal privacy aspects. In addition, technological advances and miniaturization of wearable inertial sensors made easier the collection of data for a wide range of human activities. As a consequence, the technique based on wearable-sensors has gained more attention for activity recognition as well as in many application domains including medical applications such as rehabilitation program and medical diagnosis (Jovanov et al., 2005). In the context of werable-sensors based activity recognition, the accelerometers are the most commonly used inertial sensors due to the advances in micro-electromechanical systems technology which have greatly promoted the use of accelerometers thanks to the considerable reduction in size (miniaturazation), in cost and in energy consumption. These sensors have shown satisfactory results to measure the human activities in both laboratory, clinical and free-living environment settings (Mathie et al., 2004). More specifically, the tri-axial accelerometers are more privileged (Yang and Hsu, 2010) with respect to uniaxial accelerometers.

The studied activities can be either static ones (postures) such as standing, sitting, lying or dynamic ones (movements) such as standing up, sitting down, walking, running, climbing stairs, etc. In general, the activity recognition from acceleration data is preceded by a preprocessing step of feature extraction. For example features, such as the mean, the standard deviation, the skewness, the kurtosis, etc. can be extracted from original data and used afterwards as classifier inputs (Altun et al., 2010; Ravi et al., 2005; Yang and Hsu, 2010). However, these methods are static ones as the models do not exploit the temporal dependence of the data. An alternative approach may use features such as zero-velocity crossings (ZVC) (Fod et al., 2002). A method for dynamic signal segmentation for activity recognition has been proposed in (Kozina et al., 2011); However, it has lower accuracy with transition instances in learning and testing set. Additional details on classification approaches for human activity recognition can be found in the recent reviews (Yang and Hsu, 2010; Altun et al., 2010; Preece et al., 2009; Kavanagh and Menz, 2008). Among the techniques used in the literature for the human activity classification, one can distinguish those using supervised machine learning-based approaches. These techniques provide a statistical association of a given activity feature to a possible class. For example, one can cite $K$-nearest neighbor ( $K$-NN) classification algorithm (Foerster, 1999), the naive Bayes classifier (Long et al., 2009) and the support vector machines 
(SVM) (Lau et al., 2009). For the unsupervised context, one can cite the approach based on Gaussian mixture models (GMM) (Allen et al., 2006) and the one based on hidden Markov model (HMM) (Lin and Kulić, 2011) or HMM with GMM emission probabilities (Mannini and Sabatini, 2010).

In this study, the human activities are detected over time through the segmentation of the accelerations time series. The data are measured over time during the activity of a given person, and at each time step, the three acceleration components, which we denote by $\left(y_{1}, y_{2}, y_{3}\right)$, are recorded. These data consist therefore in multidimensional time series with several regime changes over time, each regime is associated with an activity (e.g., see Figure 11). The problem of activity recognition can therefore be reformulated as the one of a joint segmentation of multidimensional time series, each segment is associated with an activity.

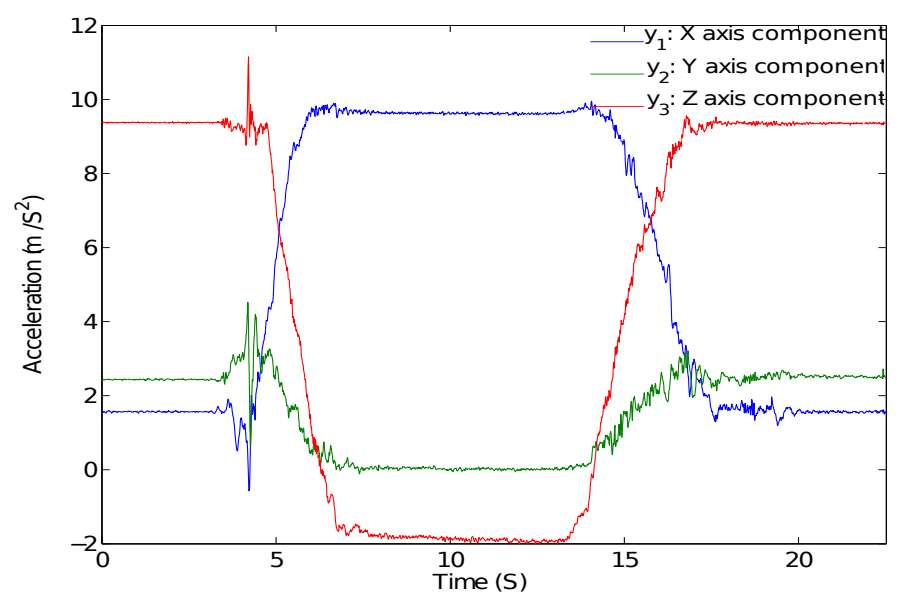

Figure 1: Example of acceleration times series.

The proposed statistical approach is dedicated to temporal segmentation by including a hidden process where the process probabilities change over time according to the most likely activity. The approach performs in an unsupervised context from raw acceleration data. More specifically, the proposed model consists in a multiple regression model governed by a discrete hidden process. The configuration of the hidden process, at each time step, corresponds to an activity described by a regression model. The hidden process configuration depends on time and the regression model parameters are time-varying according to the most likely posture. Furthermore, although 
only the acceleration measurements are available, the classes of activities being unknown, the unknown class labels are stated in the model as latent variables. In this way, we formulate an efficient and computationally tractable statistical latent data model. The resulting model is therefore a kind of latent data model which is particularly well adapted for performing unsupervised activity recognition. Let us recall that, from a statistical prospective, latent data models (Spearman, 1904) aim at representing the distribution $p(\boldsymbol{y})$ of the observed data in terms of a number of latent variables $z$. In this context of activity recognition, the observed data are the acceleration data and the latent data represent the activities (the class labels). Mixture models McLachlan and Peel. (2000) and hidden Markov models (Rabiner, 1989) are two well-known widely used examples of such models. The unsupervised learning task for the proposed approach is achieved by maximizing the observed-data log-likelihood via a dedicated iterative algorithm known as the expectation-maximization (EM) algorithm.

\section{Related work on activity recognition and time series segmenta- tion}

In this section, we briefly describe some classification approaches which are commonly used for the problem of human activity recognition. These approaches represent statistical, neural and distance-based techniques. First one can cite the naive Bayes classifier (e.g. see Mitchell (1997)) which is a supervised probabilistic classifier based on Bayes' theorem. To simplify the model estimation procedure, this classifier assumes the naive assumption that the acceleration measurements are conditionally independent given the activities. Neural approaches have also been used to perform activity recognition (Yang et al., 2008), in particular the multilayer perceptron (MLP), proposed by (Rumelhart and McClelland.,, 1986) are widely used approaches for supervied classification. The model training is performed by minimizing a cost function between the estimated and the desired network outputs (in this case the activity labels) using a gradient descent. Support vector classifiers (SVC) introduced by Vapnik (e.g., see Vapnik (1999)) are derived from statistical learning theory and are proved to be very efficient supervised classifiers. They minimize an empirical risk and at the same time attempt at finding a separating hyperplane that maximizes the margin to the classes. The $k$-Nearest Neighbor ( $k$-NN) (Cover and Hart, 1967) is a non parametric distance-based supervised classifier which is widely used because of its 
efficiency and simplicity of implementation. It classifies a data example according to a majority vote of its $k$ nearest data examples in the sense of a chosen metric, typically an Euclidean distance. A recent Dynamic Time Warping (DTW) approach with semantic attributes (SDTW) has been proposed in Pogorelc and Gams (2012) and increases the classification accuracy compared to some classical machine learning techniques for activity classification.

The classification approaches described above are supervised and require therefore a labeled collection of data to be trained. Besides, they do not exploit the temporal dependence in their model formulation as they are static approaches. The hidden Markov model (HMM) is a latent data dynamic model which is well adapted for modeling sequences and has shown its performance especially in speech recognition. It assumes that the observed sequence is governed by a hidden state (activity) sequence where the current activity depends on the previous one rather than an independence hypothesis as in the previously described classifiers. The HMM can also be used for temporal activity recognition (Mannini and Sabatini, 2010; Lin and Kulić, 2011).

In this paper, we formulate the problem of activity recognition as the one of a joint segmentation of multidimensional time series. The general problem of time series segmentation has taken great interest from different communities, including statistics, detection, signal processing, machine learning, and robotics namely activity recognition, etc. Earlier contributions in the subject were taken from a statistical point of view (McGee and Carleton, 1970; Brailovskv and Kempner,, 1992; Rabiner, 1989; Eamonn Keogh and Pazzani, 1993; Basseville and Nikiforov, 1993; Fridman, 1993; Kohlmorgen and Lemm, 2002; Nicolas Dobigeon and Scargle, 2007; Dana Kulić and Nakamura, 2008; Lin and Kulić, 2011). In (McGee and Carleton, 1970; Brailovsky and Kempner, 1992), the time series segments are modeled as several regime changes over time, each regime is assumed to be a noisy constant function and corresponds to a segment. This leads to the standard piecewise regression model proposed by (McGee and Carleton, 1970; Brailovskv and Kempner, 1992). In the piecewise regression model extended in (Brailovsky and Kempner, 1992), the data are partitioned into several segments, each segment being characterized by its mean polynomial curve and its variance. However, the parameter estimation in such a method requires the use of dynamic programming algorithm (Bellman, 1961; Stone, 1961) which may be computationally expensive especially for time series with a large number of observa- 
tions. Moreover, the standard piecewise regression model usually assumes that noise variance is uniform in all the segments (homoskedastic model). In (Basseville and Nikiforov, 1993), the problem is stated as a detection problem via hypothesis testing. This type of approach in general requires a detection threshold to reject the null hypothesis. In addition, the hypothesis testing is often used in a binary setting, the problem of multiple hypothesis testing, which is the case in this multi-class segmentation problem, is not very common while one can take each two hypotheses independently to be tested. Alternative approaches come from the machine learning community, one can cite for example the hidden Markov model (Rabiner, 1989; Lin and Kulić, 2011) which is a well-known approach that assumes that the data are arranged in an observation sequence generated by a hidden state sequence. For an activity recognition problem, each state represents an activity. The HMM can be trained in a batch-mode with a fixed number of states as well as in an online mode with varying number of states as in (Kohlmorgen and Lemm, 2002). However, in this paper, the number of activities is assumed to be fixed and the model acts in a batch mode. Another way for time series modeling is to use a hidden Markov model regression (Fridman, 1993) which is a formulation of the standard HMM into an univariate regression context. This can also be extended to a multidimensional regression setting as in (Trabelsi et al., 2011) which was also applied on time series acceleration data. Another statistical Bayesian approach that uses Gibbs sampling was also proposed for a joint segmentation of multidimensional astronomical time Series (Nicolas Dobigeon and Scargle, 2007). While this approach is non-parametric, it requires Gibbs sampling to evaluate the posterior distribution of the model and to estimate the hyperparameters of the model. This can be very limiting in terms of computational time. For the proposed approach, the posterior distribution is computed analytically and does not require sampling. In addition, while the proposed approach is parametric as in its current formulation the number of activities is given, as we shall specify it, one can use a model selection procedure to select the optimal number of activities based on some information criteria.

For the particular problem of human activity recognition, the statistical latent data models, such as standard HMMs (Lin and Kulić, 2011) or dynamic HMMs (Kohlmorgen and Lemm, 2002) have shown their performance in terms of activity recognition based on times series segmentation. Another approach uses a combined segmentation and clustering approach, and is also based on HMMs as in (Dana Kulić and Nakamura, 2008). These ap- 
proaches can be used namely in an online mode (Kohlmorgen and Lemm, 2002; Dana Kulić and Nakamura, 2008; Kulić and Nakamura, 2008). In this paper, we propose an alternative approach to HMMs in a context of a multiple regression in a batch-mode, it can be as well adapted to an online learning framework. The approach we propose to perform temporal segmentation of multivariate time series is based on an alternative to the Markov process in the HMM regression model (Fridman, 1993; Trabelsi et al., 2011). It also directly uses the raw acceleration data rather than performing feature extraction and feature selection as in (Altun et al., 2010; Ravi et al., 2005; Yang and $\mathrm{Hsu}$, 2010). In the next section, we will present the proposed model for joint segmentation of multiple time series.

\section{The multiple regression model with a hidden logistic process (MRHLP)}

Let $\mathbf{Y}=\left(\boldsymbol{y}_{1}, \ldots, \boldsymbol{y}_{n}\right)$ be a time series of $n$ multidimensional observations $\boldsymbol{y}_{i}=\left(y_{i}^{(1)}, \ldots, y_{i}^{(d)}\right)^{T} \in \mathbb{R}^{d}$ regularly observed at the time points $\mathbf{t}=\left(t_{1}, \ldots, t_{n}\right)$. Assume that the observed time series is generated by a $K$ state hidden process and let $\mathbf{z}=\left(z_{1}, \ldots, z_{n}\right)$ be the unknown (hidden) state sequence associated with the time series $\left(\boldsymbol{y}_{1}, \ldots, \boldsymbol{y}_{n}\right)$ with $z_{i} \in\{1, \ldots, K\}$. Each observation $\boldsymbol{y}_{i}$ represents an acceleration measurement and the corresponding state $z_{i}$ represents its associated activity.

The proposed approach extends the regression model with a hidden logistic process (RHLP) (Chamroukhi et al., 2009) which is concerned with univariate time series, to the multivariate case. Furthermore, the general model formulation presented in this paper includes an additional point, that is the possibility to train the polynomial dynamics with different orders rather than assuming a common order for all the polynomials.

In this way, the model offers more flexibility allowing the capture of nonlinearities between different activity acceleration data. Let us first recall that the basic univariate RHLP model is stated as follows:

$$
y_{i}=\boldsymbol{\beta}_{z_{i}}^{T} \mathbf{t}_{i}+\sigma_{z_{i}} \epsilon_{i} \quad ; \quad \epsilon_{i} \sim \mathcal{N}(0,1), \quad(i=1, \ldots, n)
$$

where $z_{i}$ is a hidden discrete-valued variable taking its values in the set $\{1, \ldots, K\}$. The variable $z_{i}$ controls the switching from one activity to another for $K$ activities at each time $t_{i}$. The vector $\boldsymbol{\beta}_{z_{i}}=\left(\beta_{z_{i} 0}, \ldots, \beta_{z_{i} p}\right)^{T}$ is the one of regression coefficients of the polynomial regression model $z_{i}$, 
$\mathbf{t}_{i}=\left(1, t_{i}, t_{i}^{2} \ldots, t_{i}^{p}\right)^{T}$ is the $p+1$ dimensional covariate vector at time $t_{i}$ and the finite integer $p$ represents the polynomial order. The RHLP model assumes that the hidden sequence $\mathbf{z}=\left(z_{1}, \ldots, z_{n}\right)$ is a hidden logistic process detailed in (Chamroukhi et al., 2009) and which will be recalled subsequently.

For the multiple regression case studied here, the model is reformulated as a set of several polynomial regression models (RHLP) for univariate data and is stated as follows:

$$
\begin{aligned}
y_{i}^{(1)} & =\boldsymbol{\beta}_{z_{i}}^{(1) T} \mathbf{t}_{i}+\sigma_{z_{i}}^{(1)} \epsilon_{i} \\
y_{i}^{(2)} & =\boldsymbol{\beta}_{z_{i}}^{(2) T} \mathbf{t}_{i}+\sigma_{z_{i}}^{(2)} \epsilon_{i} \\
\vdots & \vdots \\
y_{i}^{(d)}= & \boldsymbol{\beta}_{z_{i}}^{(d) T} \mathbf{t}_{i}+\sigma_{z_{i}}^{(d)} \epsilon_{i}
\end{aligned}
$$

where $d$ represents the dimension of the time series and the latent process $\mathbf{z}$ simultaneously governs all the univariate time series components. This allows therefore for a joint segmentation of the acceleration data over time. In (2), we have $\mathbf{t}_{i}=\left(1, t_{i}, t_{i}^{2} \ldots, t_{i}^{p_{k}}\right)^{T}$ with $p_{k}$ is the degree of the polynomial model associated with the class $z_{i}=k$. The model (2) can be rewritten in a matrix form as:

$$
\boldsymbol{y}_{i}=\mathbf{B}_{z_{i}}^{T} \mathbf{t}_{i}+\mathbf{e}_{i} \quad ; \quad \mathbf{e}_{i} \sim \mathcal{N}\left(\mathbf{0}, \boldsymbol{\Sigma}_{z_{i}}\right), \quad(i=1, \ldots, n)
$$

where $\boldsymbol{y}_{i}=\left(y_{i}^{(1)}, \ldots, y_{i}^{(d)}\right)^{T}$ is the $j$ th observation in $\mathbb{R}^{d}, \mathbf{B}_{k}=\left[\boldsymbol{\beta}_{k}^{(1)}, \ldots, \boldsymbol{\beta}_{k}^{(d)}\right]$ is a $(p+1) \times d$ dimensional matrix of the multiple regression model parameters associated with the regime (class) $z_{i}=k$ and $\Sigma_{z_{i}}$ its corresponding covariance matrix.

The probability distribution of the process $\mathbf{z}=\left(z_{1}, \ldots, z_{n}\right)$, that allows for the switching from one regression model to another, and therefore from a posture or a movement to another, is assumed to be logistic. Formally, the hidden logistic process assumes that the variables $z_{i}(i=1, \ldots, n)$, given the vector $\boldsymbol{t}=\left(t_{1}, \ldots, t_{n}\right)$, are generated independently according to the multinomial distribution $\mathcal{M}\left(1, \pi_{1}\left(t_{i} ; \mathbf{w}\right), \ldots, \pi_{K}\left(t_{i} ; \mathbf{w}\right)\right)$, where:

$$
\pi_{k}\left(t_{i} ; \mathbf{w}\right)=p\left(z_{i}=k \mid t_{i} ; \mathbf{w}\right)=\frac{\exp \left(\boldsymbol{w}_{k}^{T} \boldsymbol{v}_{i}\right)}{\sum_{\ell=1}^{K} \exp \left(\boldsymbol{w}_{\ell}^{T} \boldsymbol{v}_{i}\right)},
$$

is the logistic transformation of a linear function of the time-dependent covariate vector $\boldsymbol{v}_{i}=\left(1, t_{i}, \ldots, t_{i}^{u}\right)^{T}, \boldsymbol{w}_{k}=\left(w_{k 0}, w_{k 1}, \ldots, w_{k q}\right)^{T}$ is the $u+1$ dimensional coefficients vector associated with $\boldsymbol{v}_{i}$ and $\mathbf{w}=\left(\boldsymbol{w}_{1}, \ldots, \boldsymbol{w}_{K}\right)$. 
The RHLP process is well-adapted for capturing both abrupt and/or smooth changes of activities thanks to the flexibility of the logistic distribution. The relevance of the logistic process in terms of flexibility of transition is detailed in Chamroukhi et al. (2009).

Now consider the distribution of the observed data for the proposed model. It can be easily shown that the observation $\boldsymbol{y}_{i}$ at each time point $t_{i}$ is distributed according to the following conditional normal mixture density:

$$
p\left(\boldsymbol{y}_{i} \mid t_{i} ; \boldsymbol{\theta}\right)=\sum_{k=1}^{K} \pi_{k}\left(t_{i} ; \mathbf{w}\right) \mathcal{N}\left(\boldsymbol{y}_{i} ; \mathbf{B}_{k}^{T} \mathbf{t}_{i}, \boldsymbol{\Sigma}_{k}\right),
$$

where $\boldsymbol{\theta}=\left(\mathbf{w}, \mathbf{B}_{1}, \ldots, \mathbf{B}_{K}, \boldsymbol{\Sigma}_{1}, \ldots, \boldsymbol{\Sigma}_{K}\right)$ is the unknown parameter vector to be estimated.

\subsection{Parameter estimation by a dedicated EM algorithm}

The parameter $\boldsymbol{\theta}$ is estimated using the maximum likelihood method. As in the classic regression models, we assume that, given $\boldsymbol{t}=\left(t_{1}, \ldots, t_{n}\right)$, the $\epsilon_{i}$ are independent. This also implies the independence of $\boldsymbol{y}_{i}(i=1, \ldots, n)$ given the time vector $\mathbf{t}$. The log-likelihood of $\boldsymbol{\theta}$ for the observed data $\mathbf{Y}=$ $\left(\boldsymbol{y}_{1}, \ldots, \boldsymbol{y}_{n}\right)$ is therefore written as:

$$
\mathcal{L}(\boldsymbol{\theta} ; \mathbf{Y}, \mathbf{t})=\sum_{i=1}^{n} \log \sum_{k=1}^{K} \pi_{k}\left(t_{i} ; \mathbf{w}\right) \mathcal{N}\left(\boldsymbol{y}_{i} ; \mathbf{B}_{k}^{T} \mathbf{t}_{i}, \boldsymbol{\Sigma}_{k}\right) .
$$

The maximization of this log-likelihood cannot be performed in a closed form since it results in a complex nonlinear function due to the logarithm of the sum. However, in this context of latent data model, the expectationmaximization (EM) algorithm (McLachlan and Krishnan, 1997; Dempster et al., 1977) is particularly adapted for maximizing the log-likelihood. To derive the EM algorithm for the proposed model, we first give the complete-data loglikelihood:

$$
\mathcal{L}_{c}(\boldsymbol{\theta} ; \mathbf{Y}, \mathbf{z}, \mathbf{t})=\sum_{i=1}^{n} \sum_{k=1}^{K} z_{i k} \log \left[\pi_{k}\left(t_{i} ; \mathbf{w}\right) \mathcal{N}\left(\boldsymbol{y}_{i} ; \mathbf{B}_{k}^{T} \mathbf{t}_{i}, \boldsymbol{\Sigma}_{k}\right)\right]
$$

where $z_{i k}$ is an indicator-binary variable such that $z_{i k}=1$ if $z_{i}=k$ (i.e., when $\boldsymbol{y}_{i}$ is generated by the $k$ th regression model), and 0 otherwise. The next section presents the dedicated EM algorithm for the multiple regression model with hidden logistic process (MRHLP). 


\subsection{The dedicated EM algorithm}

The proposed EM algorithm starts with an initial parameter $\boldsymbol{\theta}^{(0)}$ and alternates between the two following steps until convergence:

E-Step. This step consists of computing the expectation of the complete-data $\log$-likelihood (7), given the observations and the current value $\boldsymbol{\theta}^{(q)}$ of the parameter $\boldsymbol{\theta}$ ( $q$ being the current iteration):

$$
\begin{aligned}
Q\left(\boldsymbol{\theta}, \boldsymbol{\theta}^{(q)}\right) & =\mathbb{E}\left[\mathcal{L}_{c}(\boldsymbol{\theta} ; \mathbf{Y}, \mathbf{t}, \mathbf{z}) \mid \mathbf{Y}, \mathbf{t} ; \boldsymbol{\theta}^{(q)}\right] \\
& =\sum_{i=1}^{n} \sum_{k=1}^{K} \tau_{i k}^{(q)} \log \pi_{k}\left(t_{i} ; \mathbf{w}\right)+\sum_{i=1}^{n} \sum_{k=1}^{K} \tau_{i k}^{(q)} \log \mathcal{N}\left(\boldsymbol{y}_{i} ; \mathbf{B}_{k}^{T} \mathbf{t}_{i}, \boldsymbol{\Sigma}_{k}\right)
\end{aligned}
$$

where:

$$
\begin{aligned}
\tau_{i k}^{(q)} & =\mathbb{E}\left[z_{i k} \mid \boldsymbol{y}_{i}, t_{i} ; \boldsymbol{\theta}^{(q)}\right]=p\left(z_{i k}=1 \mid \boldsymbol{y}_{i}, t_{i} ; \boldsymbol{\theta}^{(q)}\right) \\
& =\frac{\pi_{k}\left(t_{i} ; \mathbf{w}^{(q)}\right) \mathcal{N}\left(\boldsymbol{y}_{i} ; \mathbf{B}_{k}^{T(q)} \mathbf{t}_{i}, \mathbf{\Sigma}_{k}^{(q)}\right)}{\sum_{\ell=1}^{K} \pi_{\ell}\left(t_{i} ; \mathbf{w}^{(q)}\right) \mathcal{N}\left(\boldsymbol{y}_{i} ; \mathbf{B}_{\ell}^{T(q)} \mathbf{t}_{i}, \boldsymbol{\Sigma}_{\ell}^{(q)}\right)}
\end{aligned}
$$

is the posterior probability that $\boldsymbol{y}_{i}$ originates from the $k$ th polynomial regression model that describes the $k$ th activity.

As shown in the expression of the $Q$-function, this step simply requires the computation of the posterior probabilities $\tau_{i k}^{(q)}$.

$M$-Step. In this step, the value of the parameter $\boldsymbol{\theta}$ is updated by computing the parameter $\boldsymbol{\theta}^{(q+1)}$ maximizing the conditional expectation $Q$ with respect to $\boldsymbol{\theta}$ :

$$
\boldsymbol{\theta}^{(q+1)}=\arg \max _{\boldsymbol{\theta}} Q\left(\boldsymbol{\theta}, \boldsymbol{\theta}^{(q)}\right) .
$$

Let us denote by $Q_{\mathbf{w}}\left(\mathbf{w}, \boldsymbol{\theta}^{(q)}\right)$ the term in $Q\left(\boldsymbol{\theta}, \boldsymbol{\theta}^{(q)}\right)$ that is function of $\mathbf{w}$ and by $Q_{\boldsymbol{\theta}_{k}}\left(\boldsymbol{\theta}, \boldsymbol{\theta}^{(q)}\right)$ the term in $Q\left(\boldsymbol{\theta}, \boldsymbol{\theta}^{(q)}\right)$ that depends on $\boldsymbol{\theta}_{k}=\left(\mathbf{B}_{k}, \boldsymbol{\Sigma}_{k}\right)$, Thus:

$$
Q\left(\boldsymbol{\theta}, \boldsymbol{\theta}^{(q)}\right)=Q_{\mathbf{w}}\left(\mathbf{w}, \boldsymbol{\theta}^{(q)}\right)+\sum_{k=1}^{K} Q_{\boldsymbol{\theta}_{k}}\left(\boldsymbol{\theta}, \boldsymbol{\theta}^{(q)}\right),
$$

where:

$$
Q_{\mathbf{w}}\left(\mathbf{w}, \boldsymbol{\theta}^{(q)}\right)=\sum_{i=1}^{n} \sum_{k=1}^{K} \tau_{i k}^{(q)} \log \pi_{k}\left(t_{i} ; \mathbf{w}\right)
$$


and

$$
Q_{\boldsymbol{\theta}_{k}}\left(\boldsymbol{\theta}, \boldsymbol{\theta}^{(q)}\right)=\sum_{i=1}^{n} \tau_{i k}^{(q)} \log \mathcal{N}\left(\boldsymbol{y}_{i} ; \mathbf{B}_{k}^{T} \mathbf{t}_{i}, \boldsymbol{\Sigma}_{k}\right)
$$

for $k=1, \ldots, K$. Thus, the maximization of $Q$ with respect to $\boldsymbol{\theta}$ can be performed by separately maximizing $Q_{\mathbf{w}}\left(\mathbf{w}, \boldsymbol{\theta}^{(q)}\right)$ with respect to $\mathbf{w}$ and $Q_{\boldsymbol{\theta}_{k}}\left(\boldsymbol{\theta}, \boldsymbol{\theta}^{(q)}\right)$ with respect to $\left(\mathbf{B}_{k}, \boldsymbol{\Sigma}_{k}\right)$ for all $k=1, \ldots, K$. Maximizing $Q_{\boldsymbol{\theta}_{k}}\left(\boldsymbol{\theta}, \boldsymbol{\theta}^{(q)}\right)$ with respect to $\mathbf{B}_{k}$ consists of solving the weighted least-squares problem where the weights are the posterior probabilities $\tau_{i k}^{(q)}$. The solution to this problem is obtained in a closed form from the so-called normal equations and is given by:

$$
\mathbf{B}_{k}^{(q+1)}=\left(\mathbf{X}^{T} \mathbf{W}_{k}^{(q)} \mathbf{X}\right)^{-1} \mathbf{X}^{T} \mathbf{W}_{k}^{(q)} \mathbf{Y}
$$

where $\mathbf{W}_{k}^{(q)}$ is an $n \times n$ diagonal matrix of weights whose diagonal elements are the posterior probabilities $\left(\tau_{1 k}^{(q)}, \ldots, \tau_{n k}^{(q)}\right)$ and $\mathbf{X}$ is the $n \times(p+1)$ regression matrix. Maximizing $Q_{\boldsymbol{\theta}_{k}}\left(\boldsymbol{\theta}, \boldsymbol{\theta}^{(q)}\right)$ with respect to $\boldsymbol{\Sigma}_{k}$ is a weighted variant of the problem of estimating the variance of a multivariate Gaussian density. The problem can be solved in a closed form and the solution is given by:

$$
\Sigma_{k}^{(q+1)}=\frac{1}{\sum_{i=1}^{n} \tau_{i k}^{(q)}}\left(\mathbf{Y}-\mathbf{X B}_{k}^{(q+1)}\right)^{T} \mathbf{W}_{k}^{(q)}\left(\mathbf{Y}-\mathbf{X B}_{k}^{(q+1)}\right)
$$

The maximization of $Q_{\mathbf{w}}\left(\mathbf{w}, \boldsymbol{\theta}^{(q)}\right)$ with respect to $\mathbf{w}$ is a multinomial logistic regression problem weighted by $\tau_{i k}^{(q)}$ which we solve with a multiclass Iterative Reweighted Least Squares (IRLS) algorithm (Green, 1984; Chen et al., 1999; Krishnapuram et al., 2005; Chamroukhi et al., 2009).

The time complexity of the E-step of this EM algorithm is of $\mathcal{O}(K n)$. The calculation of the regression coefficients in the M-step requires the computation and the inversion of the square matrix $\mathbf{X}^{T} \mathbf{X}$ which is of dimension $d \times(p+1)$, and a multiplication by the observation sequence of length $m$ which has a time complexity of $\mathcal{O}\left(d^{2}(p+1)^{2} n\right)$. In addition, each IRLS loop requires an inversion of the Hessian matrix which is of dimension $2 \times(K-1)$. The complexity of the IRLS loop is then approximatively of $\mathcal{O}\left(I_{\text {IRLS }} K^{2}\right)$ where $I_{\mathrm{IRLS}}$ is the average number of iterations required by the internal IRLS algorithm. The proposed algorithm has therefore a time complexity of $\mathcal{O}\left(I_{\mathrm{EM}} I_{\mathrm{IRLS}} K^{3} d^{2} p^{2} n\right)$, where $I_{\mathrm{EM}}$ is the number of iterations of the EM algorithm. The pseudo code 1 summarizes one run of the proposed EM algorithm. 


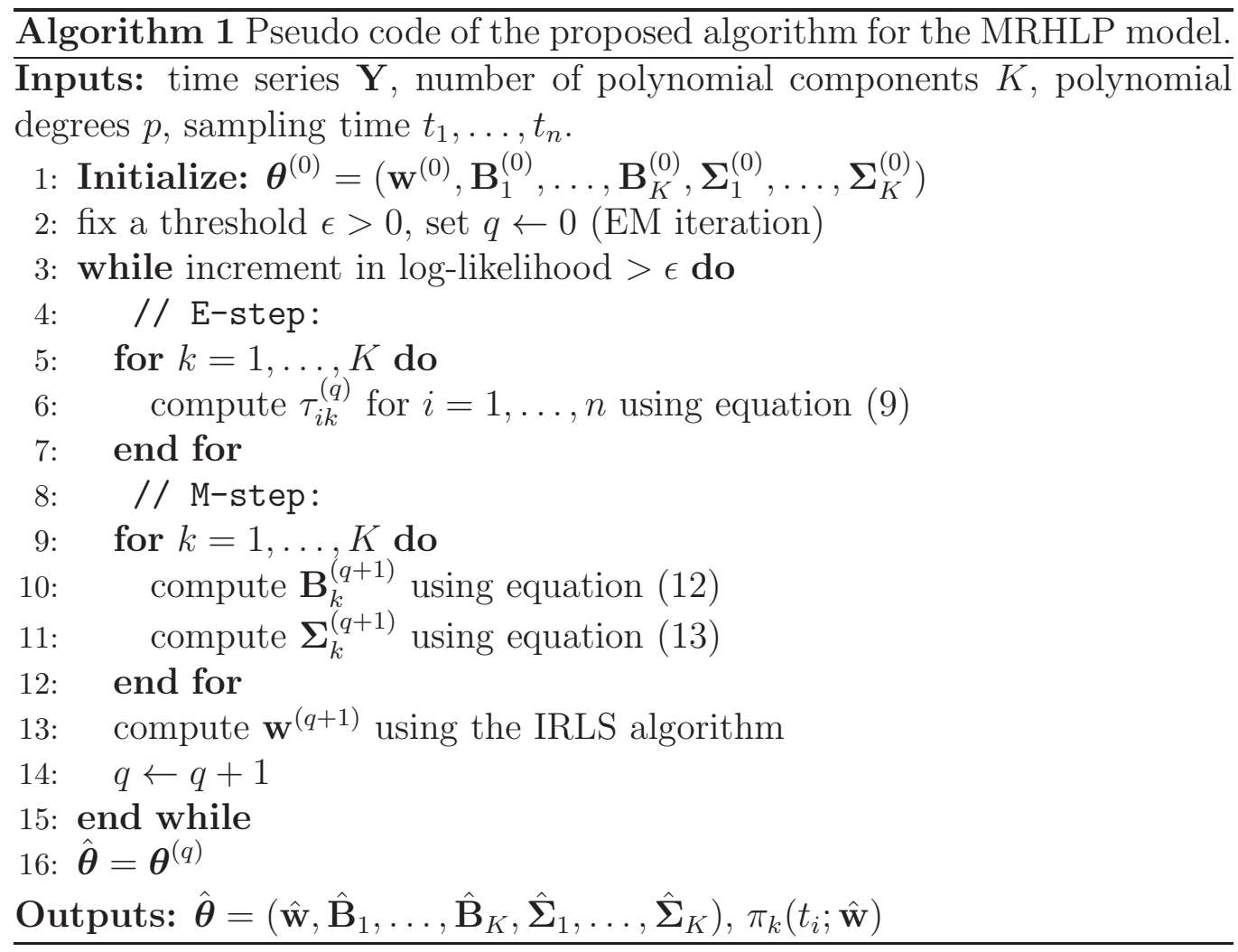

Once the model parameters are estimated by the EM algorithm, the time series segmentation can be obtained by computing the estimated label $\hat{z}_{i}$ of the polynomial regime generating each measurement $\boldsymbol{y}_{i}$. This can be achieved by maximizing the following probability, that is:

$$
\hat{z}_{i}=\arg \max _{1 \leq k \leq K} \pi_{k}\left(t_{i} ; \hat{\mathbf{w}}\right), \quad(i=1, \ldots, n) .
$$

Note that when the logistic model (44) for $\pi_{k}$ is stated with one dimensional variable (i.e., if the probabilities $\pi_{k}$ are computed with a dimension $u=1$ of $\left.\boldsymbol{w}_{k}(k=1, \ldots, K)\right)$, applying this rule guarantees that the time series are segmented into contiguous segments.

\subsection{Selecting the number of activities}

The proposed unsupervised approach does not require any annotation of the raw acceleration data by experts to learn the model parameters. However, in its current formulation, we assume that the number of activities is known 
and therefore supplied by the user (in practice this can for example be given by an expert). In a general use of the proposed model, namely when mining amount of data for which no information regarding the activities is available, the optimal value of $K$ can be estimated automatically from the data by using for example the Bayesian Information Criterion (BIC) (Schwarz, 1978) which is a penalized likelihood criterion, defined as:

$$
\operatorname{BIC}(K, p, u)=\mathcal{L}(\hat{\boldsymbol{\theta}})-\frac{\nu_{\boldsymbol{\theta}} \log (n)}{2},
$$

where $\nu_{\boldsymbol{\theta}}=\nu_{\boldsymbol{\theta}}=K(p+4)-2$ is the number of free parameters of the model and $\mathcal{L}(\hat{\boldsymbol{\theta}})$ is the observed-data log-likelihood obtained at convergence of the EM algorithm.

\section{Experimental study}

In this section, the application of the proposed approach is illustrated on real time series of acceleration data measured during human activities. The model performances are compared to those obtained with alternative activity recognition approaches. The evaluation criterion is the error segmentation (classification) between the obtained segmentation and the ground truth. In the following subsection, we describe the experimental set up.

\subsection{Experimental Setup}

The sensors used for data acquisition consisted of three MTx 3-DOF inertial trackers developed by Xsens Technologies (Enschede, 2009). Each

MTx unit includes a tri-axial accelerometer measuring the acceleration in the 3 -d space (with a dynamic range of $\pm 5 g$ where $g$ represents the gravitational constant). The sensor's placements is chosen to represent the human body motion while guaranteeing less constraint and better comfort for the wearer as well as its security. The sensors were placed at the chest, the right thigh and the left ankle respectively as shown in Figure 2. As shown in Bouten et al. (1997), points near the hip and torso exhibit a $6 g$ range in acceleration. Our experiences show also that the measured ankle-sensor accelerations during the different activities do not excceed the limit of $\pm 5 \mathrm{~g}$. The sampling frequency is set to $25 \mathrm{~Hz}$, which is sufficient and larger than $20 \mathrm{~Hz}$ the required frequency to assess daily physical activity (Bouten et al., 1997). The sensors were fixed on the subjects with the help of an assistant before the begining of the measurement operation. Sensors placement is chosen to represent 
predominantly upper-body activities such as standing up, sitting down, etc. and predominantly lower body activities such as walking, stair ascent, stair descent, etc. To secure each MTx unit in place, specific straps are used. This combination allows for efficient inter-subject transfer. The MTx units are connected to a central unit called Xbus Master which is attached to the subject's belt. Raw acceleration data are collected over time when performing the activities and the data transmission between units and the receiver is carried out through a Bluetooth wireless link.

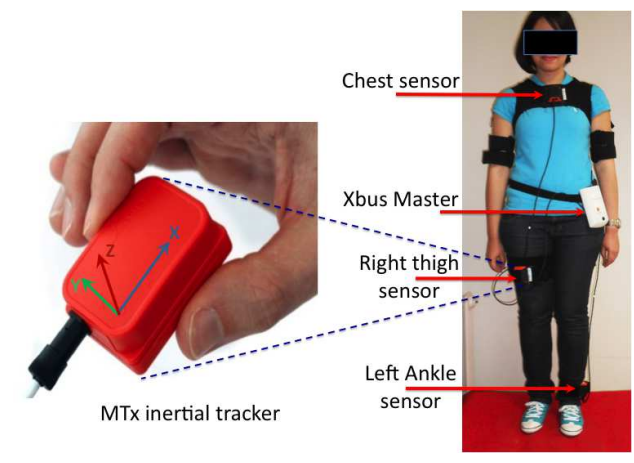

Figure 2: MTx-Xbus inertial tracker and sensors placement

The experiments were conducted at the LISSI Lab/University of Paris-Est Créteil (UPEC) by six different healthy subjects of different ages (who are not the researchers) in the office environment. In order to gather representative dataset, the recruited volunteers subjects have been chosen in a given marge of age (25-30) and weight (55-70) kg. Activity labels were estimated by an independent operator. Data are stored on a file and acceleration signals are analyzed using MATLAB software. Twelve activities and transitions were studied and are shown in Table 1 and some of these activities are illustrated on Figure 3 . 


\begin{tabular}{|c|c|}
\hline Activity reference & Description \\
\hline $\mathrm{A}_{1}$ & Stair descent \\
\hline $\mathrm{A}_{2}$ & Standing \\
\hline $\mathrm{A}_{3}$ & Sitting down \\
\hline $\mathrm{A}_{4}$ & Sitting \\
\hline $\mathrm{A}_{5}$ & From sitting to sitting on the ground \\
\hline $\mathrm{A}_{6}$ & Sitting on the ground \\
\hline $\mathrm{A}_{7}$ & Lying down \\
\hline $\mathrm{A}_{8}$ & Lying \\
\hline $\mathrm{A}_{9}$ & From lying to sitting on the ground \\
\hline $\mathrm{A}_{10}$ & Standing up \\
\hline $\mathrm{A}_{11}$ & Walking \\
\hline $\mathrm{A}_{12}$ & Stair ascent \\
\hline
\end{tabular}

Table 1: Description of the considered activities.

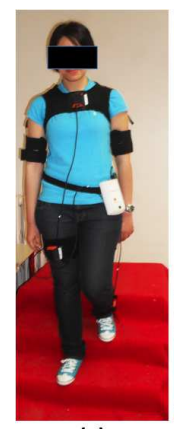

(a)

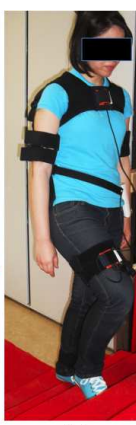

(b)

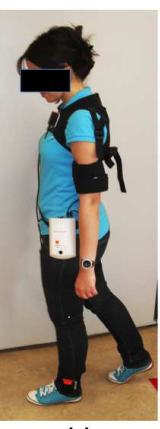

(c)

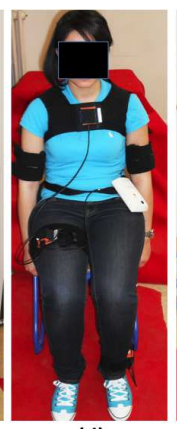

(d)

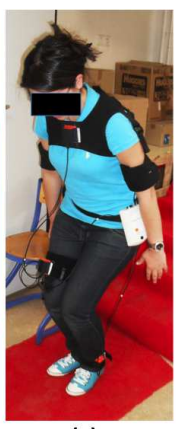

(e)

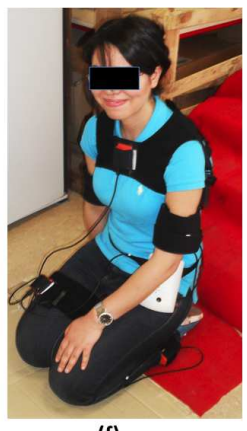

(f)

Figure 3: Examples of some considered activities: a) Stair descent, b) Stair ascent, c) Walking, d) Sitting, e) Standing up, f) Sitting on the ground.

The activities were chosen to have an appropriate representation of everyday activities involving different parts of the body. The recognized activities and transition differ in duration and intensity level. The subjects were asked to perform the activities in their own style and were not restricted on how the activities should be performed but only with the sequential activities order. Note that the activities $A_{3}, A_{5}, A_{7}, A_{9}$ and $A_{11}$ represent dynamic transitions between static activities.

For the three sensor units, each unit being a tri-axial accelerometer, a 9dimensional acceleration time series are recorded overtime for each activity. The time series present regime changes over time, in which each regime is 
associated to an activity. Figure 1illustrates an example of three acceleration data measured on the chest inertial sensor for the three activities' scenario: standing - transition (standing to sitting) - sitting - (transition) sitting to standing - standing.

\subsection{Results and discussion}

In this section, we present and discuss the results obtained by applying the EM algorithm of the proposed MRHLP model to the temporal segmentation of the acceleration time series. The data are acquired according to the experimental setup described previously. First, results are given on some activities for illustration and then the numerical results are given for the whole activities according to an experimental protocol which will be described subsequently.

Consider the activities illustrated in Figure 1 and consider for that an MRHLP model to automatically recognize the three activities. Note that in this scenario, we have two static activities with a transitory dynamic activity between sitting and standing $(K=3)$. Figure 4 shows the results obtained for both the MRHLP model and the HMM model. 


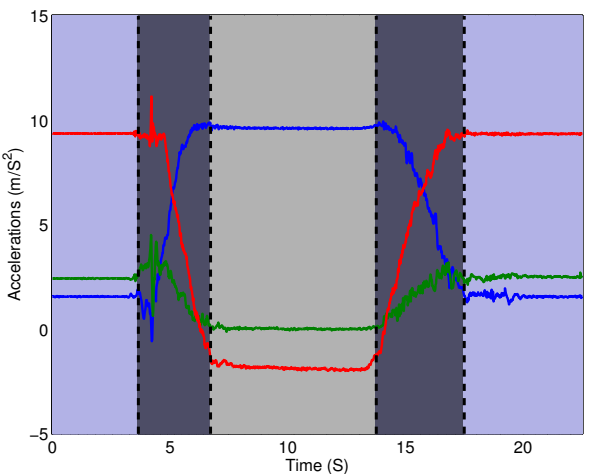

(a)

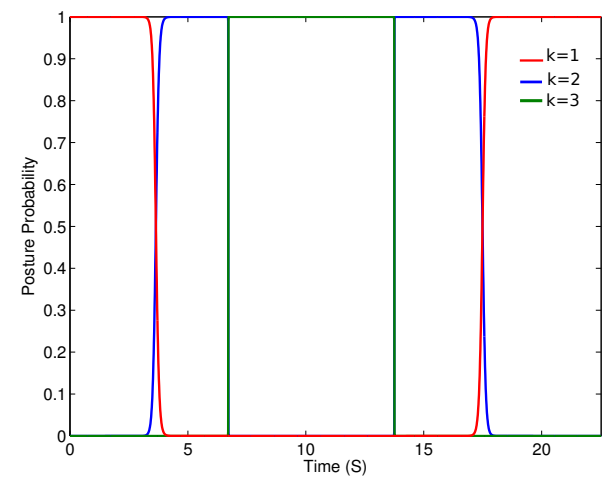

(c)

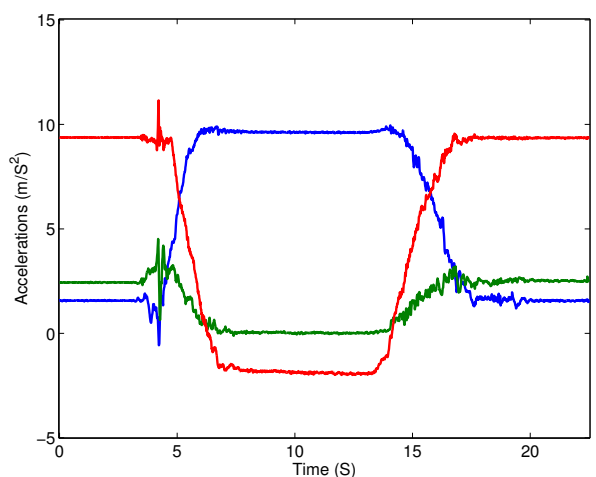

(b)

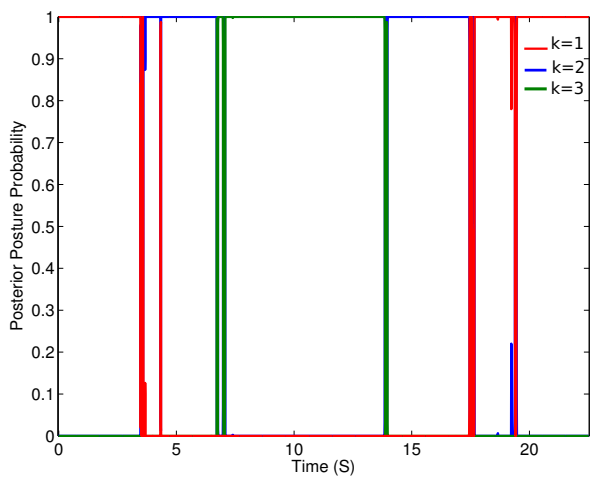

(d)

Figure 4: Results obtained by applying the proposed MRHLP model (left) and the HMM approach (right) on the acceleration time series measured from human activity scenario shown in Figure 1 with $k=1$ : standing $k=2$ : transition between standing and sitting, and $k=3$ : sitting.

It can be observed on Figure 4 (c) that the estimated probabilities of the logistic process that govern the switching from one activity to another over time correspond to an accurate segmentation of the acceleration times series. The acceleration data, segmented automatically, are shown in Figure 4 (a). The recognized activities are represented with different colors. Moreover, the flexibility of the logistic process allows to get smooth probabilities in particular for the transitions before $5 \mathrm{~s}$ and after $15 \mathrm{~s}$. This can be beneficial in practice especially to decide whether or not a decision can be made about the activity by fixing a threshold, for example 0.5 .

On the other hand, Figure 4 (d) shows the posterior activity probabilities estimated by a HMM in which a standard homogeneous Markov process gov- 
erns the latent activity sequence. We clearly observe segmentation errors in the transitory phases and even when the person maintains the same activity (see the standing activity before the instant $20 \mathrm{~s}$ ).

We note that we can go more in detail in analyzing the activities by studying the transitions between activities. For the previous scenario, if the aim is to have more precise information on the transitions, for example to know when we are still close to the current activity or the one after, or for a more general analysis, this can be achieved by adding to the model another activity to be retrieved. This can be observed on the results presented on Figure 5 and which are obtained for a scenario of four activities rather than a three activities scenario as described previously. The scenario is: standing - (transition) standing to sitting - sitting - (transition) sitting to standing - standing. In this case, the transitory phase is analyzed as two pseudo transitions. The first transition is the one close to the previous activity and the second one is the one close to the activity to which we are going on. It can be clearly observed that the main activities still correctly segmented. Furthermore, within the transitory phases shown previously in Figure 4 (a), additional segmentation is provided according to which can be seen as "pseudo" transition within each transition (see Figure 5 (a) and (c) around $5 \mathrm{~s}$ and $15 \mathrm{~s}$ ). We can observe for example that the person seems to spend more time in standing up than in sitting down due to the first step of the transition to stand up which takes more time than the second step. The HMM approach, as shown in Figure 5 provides a less satisfactory results than the proposed approach. 


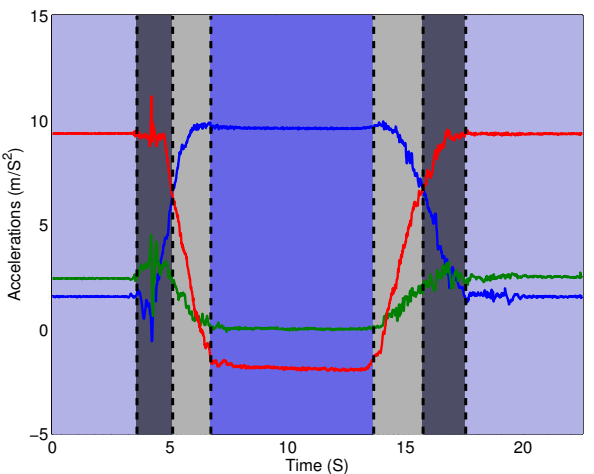

(a)

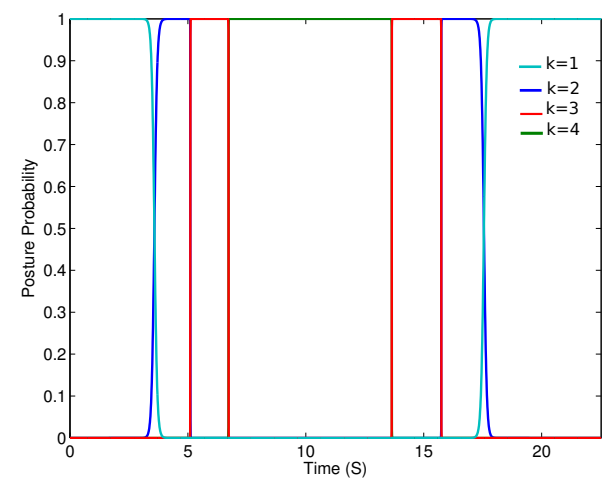

(c)

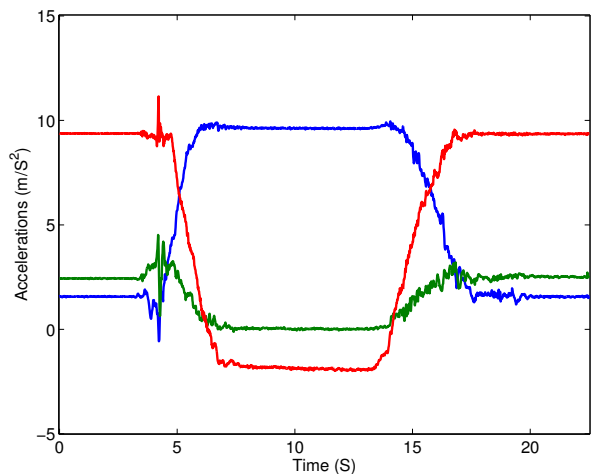

(b)

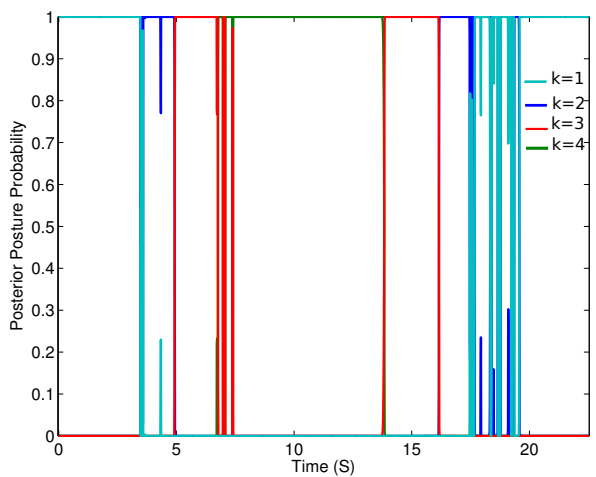

(d)

Figure 5: Results obtained by applying the proposed MRHLP model (left) and the HMM approach (right) on the acceleration time series measured from human activity scenario shown in Figure 1 by considering four activities with $k=1$ : standing $k=2$ : first pseudo transition between standing and sitting, $k=3$ : second pseudo transition between standing and sitting, and $k=4$ : standing.

The results shown previously (see Figures 4, 5) are obtained using only one accelerometer attached to the right thigh and providing 3-d raw acceleration times series. In Figure 6, we illustrate the fact that, in practice, the use of the three sensors fixed at the chest, thigh and ankle, slightly improves the segmentation (both for the MRHLP and the HMM). In particular, the probabilities of the MRHLP become more close to one for the transitions, as shown in Figure 6 (c), in comparison to the previous results shown on Figures 4 (c) and 5 (c). Figure 6 (d) also shows that the segmentation is improved for the HMM compared to the case based on a single sensor (see Figure 5 (d)). Quantitative study regarding the sensors selection has been 
studied in Trabelsi et al. (2011) and has also shown that using three sensors can improve the segmentation error (about $5 \%$ ).

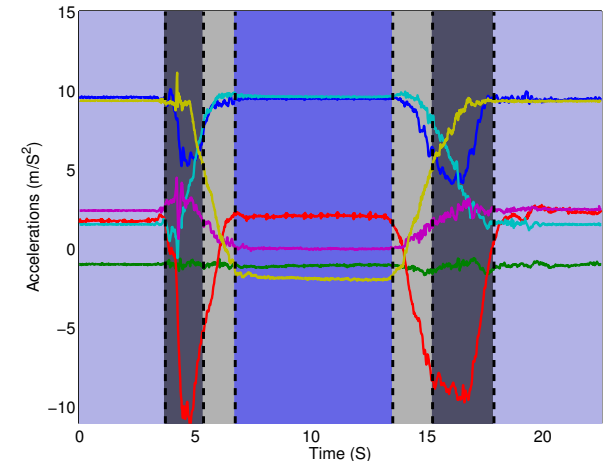

(a)

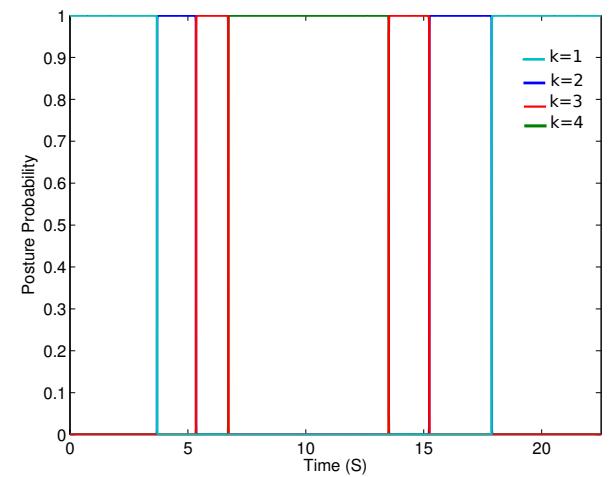

(c)

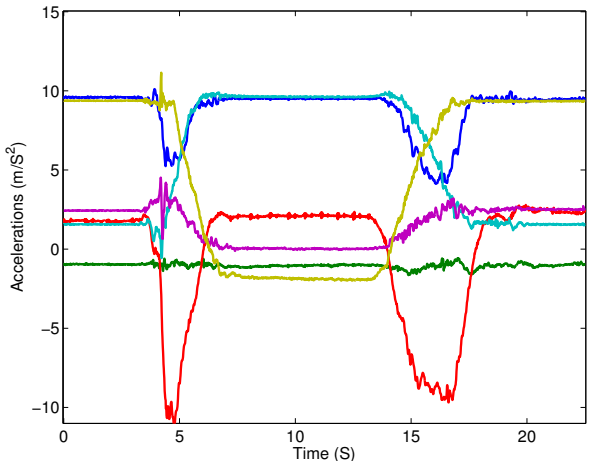

(b)

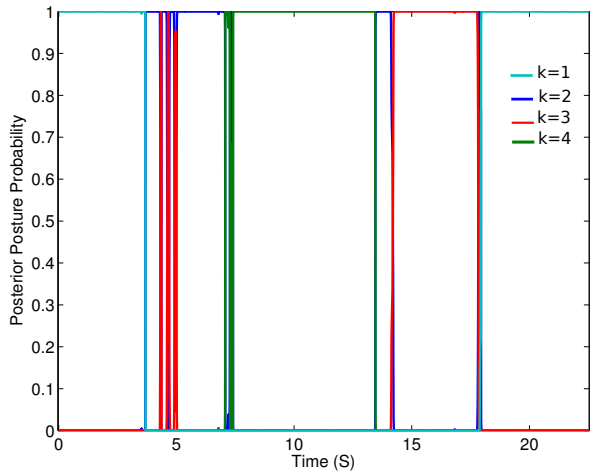

(d)

Figure 6: Results obtained by applying the proposed MRHLP model (left) and the HMM approach (right) on the whole 9-d acceleration time series measured from human activity scenario considered previously with $k=1$ : standing $k=2$ : first pseudo transition between standing and sitting, $k=3$ : second pseudo transition between standing and sitting, and $k=4:$ standing.

Now we consider the twelve activities in an experimental protocol to evaluate and quantify the performance of the MRHLP approach proposed in Section 3. The automatic segmentation of the human activity is carried out by using the 3-d raw acceleration time series. The acceleration time series data, as described previously, contain recordings of six healthy volunteers performing the twelve activities described in Table1. Each person performs the same sequence of activities. The training was done on each data sequence for each subject. To judge the performance of the proposed approach, we perform 
comparisons to the well-known static and dynamic classification approaches such as the Naive Bayes classifier, K-NN, SVM, MLP (Altun et al., 2010; Yang and Hsu, 2010; Yang et al., 2008; Preece et al., 2009) which are static supervised classification techniques and the HMM (Lin and Kulić, 2011) which is a standard temporal segmentation approach. We note that for the supervised static approaches and for the unsupervised dynamic HMM model, we used the same number of classes (states) as for the proposed approach, that is 12 activities; the transitions (i.e., A5 and A9) were defined as separated states. The dataset were divided into a training set and a test set according to a 5-fold cross validation procedure. For the supervised approaches, the classifiers were trained in a supervised way by supplying the true class labels (ground truth) in addition to the acceleration data. Then, in the test step, the class labels obtained for the test data are directly matched to the ground truth and the classification error rate is computed. While for both the HMM approach and the proposed unsupervised MRHLP model, the models are trained in an unsupervised way from only the acceleration data, the class labels are not considered in the training, they are only used afterwards to evaluate the classifier. In the test step, as the approaches act in an unsupervised way, the class labels obtained for the test data are matched to the true labels (ground truth) by evaluating all the possible matchings; the matching providing the minimum classification error rate is selected.

We notice that a main advantage of the proposed unsupervised approach is that it does not require preprocessing, the model parameters being learned in an unsupervised way from the acquired unlabelled raw acceleration data. In general, the activity recognition are two-fold as they are preceded by a preprocessing step of feature extraction from the raw acceleration data, the extracted features are then classified (Altun et al., 2010; Ravi et al., 2005; Yang and $\mathrm{Hsu}$, 2010). However, the feature extraction step may itself require implementing additional models or routines, well-established criteria or additional expertise to extract/select optimal features. Furthermore, the feature extraction step may also require an additional computational cost which can be penalizing in particular for a perspective of real time applications.

The results obtained with the different approaches are given in Table 2 . 


\begin{tabular}{|l||c|}
\hline Model & Correct segmentation (classification) rate (\%) \\
\hline \hline Naive Bayes & 80.64 \\
\hline MLP & 83.15 \\
\hline SVM & 88.10 \\
\hline$K-$ NN & 95.89 \\
\hline HMM & 84.16 \\
\hline \hline MRHLP & 90.3 \\
\hline
\end{tabular}

Table 2: Correct segmentation (classification) rates (\%) obtained with the different classifiers.

We can observe that all the correct classification rates are greater than $80 \%$. However, $K$-NN approach which provides the best results $(95.8 \%)$ requires a significant computation time due to the computation of the distances between the considered acceleration observation and all the other observation. On the other hand, the proposed approach only needs the computation of the (posterior) activity probabilities given an observation. It can also be observed that the proposed unsupervised dynamic model provides more accurate segmentation results compared to the supervised static classifiers (Naive Bayes, MLP and SVM). This can be attributed to the fact that the model, by including the time as an intrinsic variable, fits more the temporal acceleration data. While the HMM model is also a dynamic model for time series modeling, it can be observed that it does not outperforms the proposed MRHLP approach. Figures 7 and 8 show the results obtained from the proposed approach and their comparison with the ground truth segmentation. 

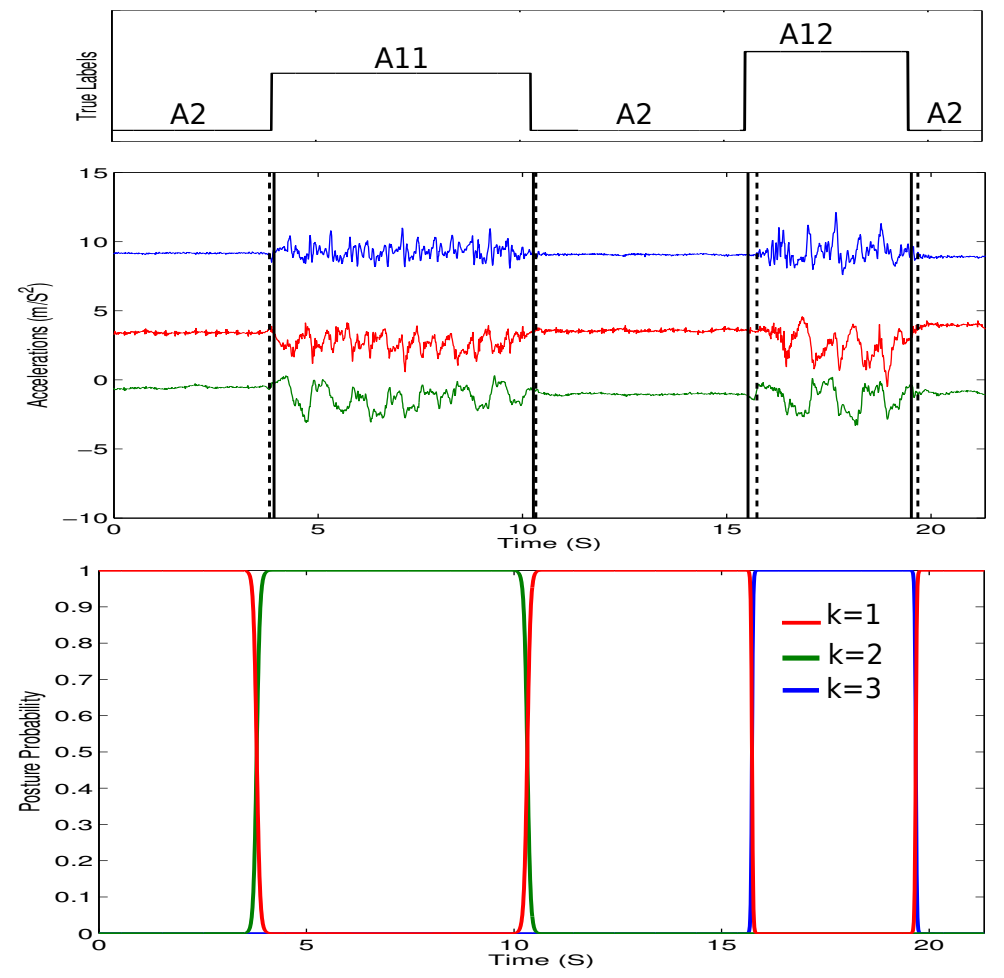

Figure 7: MRHLP segmentation results for the scenario : Standing $A_{2}(k=1)$ - Walking $\mathrm{A}_{11}(\mathrm{k}=2)$ - Standing $\mathrm{A}_{2}(\mathrm{k}=1)$ - Stair ascent $\mathrm{A}_{12}(\mathrm{k}=3)$ - Standing $\left.\mathrm{A}_{2}(\mathrm{k}=1)\right)$ with (top) the true labels, (middle) the times series and the actual segments in bold line and the estimated segments in dotted line, and (bottom) the logistic probabilities. 

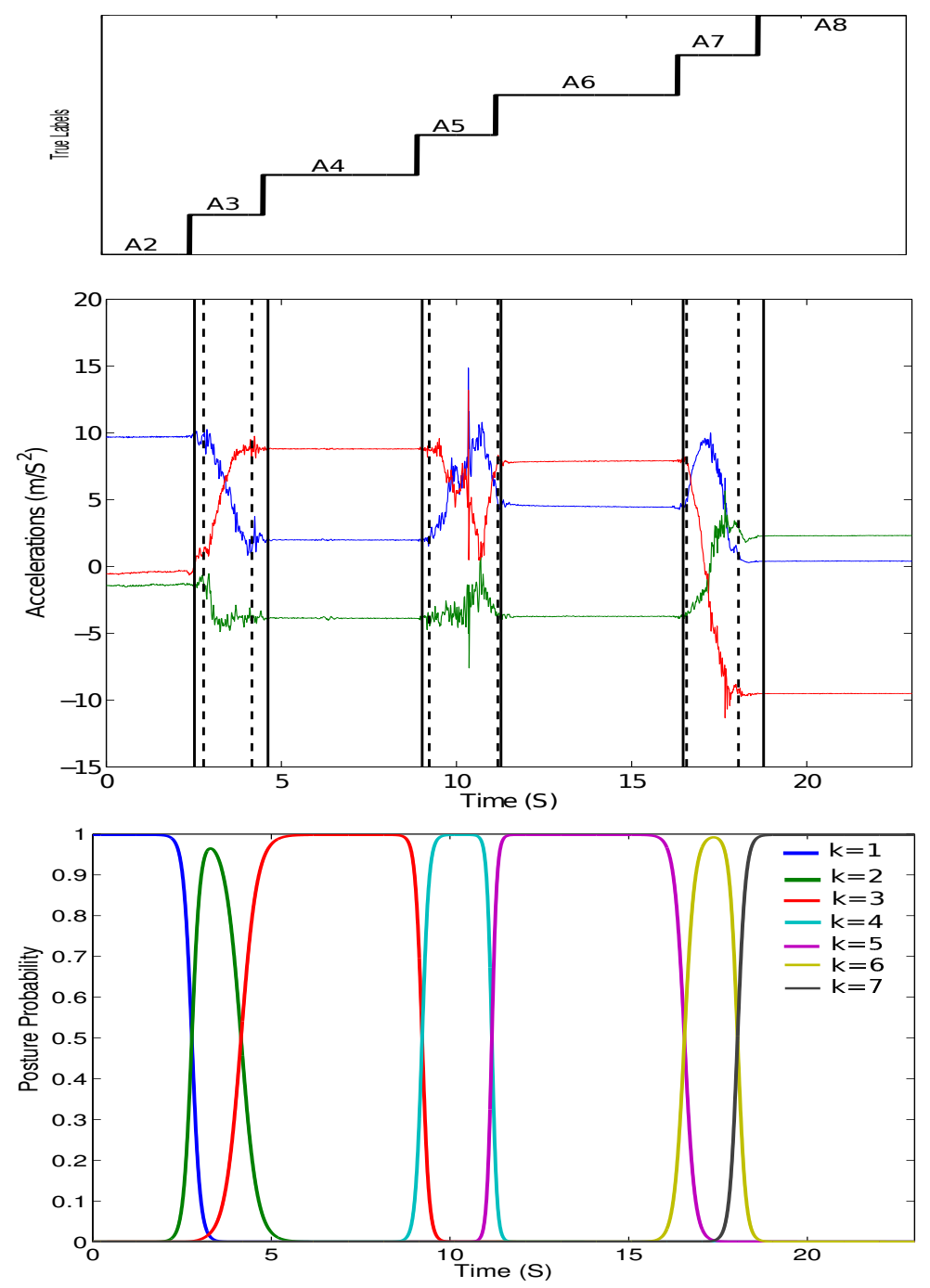

Figure 8: MRHLP segmentation results for the scenario: Standing $A_{2}(k=1)$ - Sitting down $A_{3}(k=2)$ - Sitting $A_{4}(k=3)$ - From sitting to sitting on the ground $A_{5}(k=4)$ - Sitting on the ground $\mathrm{A}_{6}(\mathrm{k}=5)$ - Lying down $\mathrm{A}_{7}(\mathrm{k}=6)$ - Lying $\mathrm{A}_{8}(\mathrm{k}=7)$ with (top) the true labels, (middle) the times series and the actual segments in bold line and the estimated segments in dotted line, and (bottom) the logistic probabilities.

For the scenario of these activities, we give the confusion matrix in Table 3. It can be observed that the confusions of the classifier occur in the regions of transitions between the activities. This can also be observed on the class probabilities where at these regions the probability becomes not very close 


\begin{tabular}{|c|c|c|c|c|c|c|c|c|}
\hline & \multicolumn{7}{|c|}{ Obtained classes } \\
\hline & & $\mathrm{A} 2$ & A3 & $\mathrm{A} 4$ & A5 & $\mathrm{A} 6$ & $\mathrm{~A} 7$ & A8 \\
\hline \multirow{7}{*}{$\begin{array}{l}\text { True } \\
\text { classes }\end{array}$} & A2 & 245 & 7 & 0 & 0 & 0 & 0 & 0 \\
\hline & A3 & 0 & 210 & 0 & 0 & 0 & 0 & 0 \\
\hline & A4 & 0 & 32 & 407 & 1 & 0 & 0 & 0 \\
\hline & A5 & 0 & 0 & 0 & 225 & 0 & 0 & 0 \\
\hline & $\mathrm{A} 6$ & 0 & 0 & 0 & 20 & 481 & 19 & 0 \\
\hline & $\mathrm{A} 7$ & 0 & 0 & 0 & 0 & 0 & 224 & 6 \\
\hline & $\mathrm{A} 8$ & 0 & 0 & 0 & 0 & 0 & 0 & 424 \\
\hline
\end{tabular}

Table 3: Confusion Matrix

to one.

These misclassification errors can be attributed to the fact that the transitions here are similar the problem of class overlap in the case of multidimensional data classification problem. However, the HMM approach, as it can be seen for example on the posterior classes probabilities shown in Figure 4(d), confusions can occur even within a homogeneous part of the class that is not necessarily near the transition (see the standing activity before the instant $20 \mathrm{~s}$ ).

The confusion matrix for the whole experiments that includes the twelve activities is given in Table 4 . Table 5 gives the corresponding false positive and false negative error rates. We can observe from the confusion matrix that the confusions occur especially between successive activities. We can also observe that the basic activities such as $\mathrm{A}_{12}$ and $\mathrm{A}_{4}$ are more easy to detect than transitions like $\mathrm{A}_{3}$.

We also evaluated the effect of the used sensors on the classification accuracy. We therefore performed the same experiments that involve the same activities by using different combinations of the sensors. As it can be seen on Table 6, the best results are obtained when the three sensors are used. This is also confirmed on Figure 5(c) and Figure 6(c) that adding sensors improves the estimation of the model since the provided activity probabilities are more precise as they are more in concordance with the true activities.

We note that all the approaches require a training step, except the $k$ $\mathrm{NN}$ which is a direct classification approach. However, the $k$-NN classifier is the more time-consuming in the test step (about 5 seconds for a single sequence). All the other approaches are not time-consuming in the test step. In the training step, the proposed algorithm and the one for the standard 
Obtained classes

\begin{tabular}{|c|c|c|c|c|c|c|c|c|c|c|c|c|c|}
\hline & & A1 & A2 & A3 & $\mathrm{A} 4$ & A5 & $\mathrm{A} 6$ & A7 & A8 & A9 & A10 & A11 & $\overline{\mathrm{A} 12}$ \\
\hline & $\overline{\mathrm{A} 1}$ & 2356 & 122 & 13 & 0 & 0 & 0 & 0 & 0 & 0 & 0 & 0 & 0 \\
\hline & A2 & 254 & 10710 & 612 & 392 & 230 & 134 & 0 & $\overline{0}$ & $\overline{0}$ & $\overline{0}$ & 0 & 0 \\
\hline & A3 & 0 & 184 & 1056 & 193 & 0 & 0 & 0 & 0 & 0 & 0 & 0 & 0 \\
\hline & A4 & 0 & 20 & 92 & 2397 & 31 & 0 & 0 & 0 & 0 & 0 & 0 & 0 \\
\hline & A5 & 0 & 17 & 0 & 33 & 1486 & 30 & 0 & 0 & 0 & 0 & 0 & 0 \\
\hline Trure & $\mathrm{A} 6$ & 0 & 12 & 0 & 0 & 64 & 5145 & 25 & 19 & 98 & 62 & 0 & 0 \\
\hline classes & A7 & 0 & 0 & 0 & 0 & 0 & 32 & 1688 & 79 & 26 & 0 & 0 & 0 \\
\hline & A8 & 0 & 0 & 0 & 0 & 0 & 68 & 123 & 2622 & 127 & 0 & 0 & 0 \\
\hline & A9 & 0 & 0 & 0 & 0 & 0 & 07 & 11 & 06 & 1355 & 13 & 0 & 0 \\
\hline & A10 & 0 & 0 & 0 & 0 & 0 & 12 & 0 & 0 & 34 & 924 & 20 & 0 \\
\hline & A11 & 0 & 0 & 0 & 0 & 0 & 0 & 0 & 0 & 21 & 58 & 3035 & 22 \\
\hline & A12 & 0 & 0 & 0 & 0 & 0 & 0 & 0 & 0 & 0 & 64 & 421 & 2395 \\
\hline
\end{tabular}

Table 4: Confusion Matrix for all the data set.

\begin{tabular}{|c|c|c|c|c|c|c|c|c|c|c|c|c|}
\hline Activity & A1 & A2 & A3 & A4 & A5 & A6 & A7 & A8 & A9 & A10 & A11 & A12 \\
\hline FP rate & 9.7 & 3.2 & 40.4 & 20.4 & 17.9 & 5.2 & 8.6 & 3.8 & 18.4 & 17.5 & 12.6 & 0.9 \\
\hline FN rate & 5.4 & 13.1 & 26.3 & 5.6 & 5.1 & 5.1 & 7.5 & 10.8 & 2.65 & 6.6 & 3.2 & 16.8 \\
\hline
\end{tabular}

Table 5: False positive and false negative error rates for the MRHLP model

\begin{tabular}{|l|c|}
\hline Sensors & Correct classification rate \\
\hline Chest, thigh, ankle & $90.3 \%$ \\
\hline Chest, ankle & $83 \%$ \\
\hline Chest, thigh & $83.4 \%$ \\
\hline Thigh, ankle & $84 . \%$ \\
\hline
\end{tabular}

Table 6: Classification results obtained with the MRHLP model according to the considered sensors

unsupervised HMM have a quasi-identical computing time and both are more fast than the MLP and the SVM. We also note that while the Naive Bayes is easy to train, it is outperformed by all the other approaches.

In summary, the proposed approach provides a statistical well established background with very encouraging performance for automatic segmentation of human activity. The flexibility of the model allows an accurate and efficient recognition of standard activities as well as transitory activities. 


\section{Conclusion and future work}

In this paper, the problem of temporal activity recognition from acceleration data is reformulated as the one of unsupervised learning of a specific statistical latent process model for the joint segmentation of multivariate time series. The main advantages of this statistical approach is that it directly uses the raw acceleration data and performs in an unsupervised context which can be beneficial in practice. For example, this may be helpful for avoiding the investments in labelling flows of acceleration data or in finding adapted preprocessing feature extraction approaches. Moreover, the model formulation explicits the switching from one activity to another during time through a flexible logistic process which is also particularly well adapted for abrupt or smooth transitions. Furthermore, the expectation-maximization algorithm offers a stable efficient optimization tool to learn the model. The proposed MRHLP approach is applied on a real-world activity recognition problem based on multidimensional acceleration time series measured using body-worn accelerometers. The approach has shown very encouraging results compared to alternative models for activity recognition. In its current formulation, the proposed algorithm runs in a batch-mode and requires the entire data sequence to be presented. A perspective of this work is to train the proposed model with an online Expectation-Maximization (EM) algorithm (as in Cappé and Moulines (2009)) for a real-time use perspective. Future work will also concern the use of other non-linear models to describe each activity signal rather than polynomial bases. This may improve in particular the representation of each activity. Then, another extension may consist int integrating the model into a Bayesian non-parametric model which will be useful for any kind of complex activities and in which the number of activities will not have to be fixed.

\section{References}

Allen, F. R., Ambikairajah, E., Lovell, N. H., Celler, B. G., Oct. 2006. Classification of a known sequence of motions and postures from accelerometry data using adapted Gaussian mixture models. Physiological Measurement 27 (10), 935-951.

Altun, K., Barshan, B., Tuncel, O., October 2010. Comparative study on classifying human activities with miniature inertial and magnetic sensors. Pattern Recognition 43, 3605-3620. 
Aminian, K., Robert, P., Buchser, E., Rutschmann, B., Hayoz, D., Depairon, M., May 1999. Physical activity monitoring based on accelerometry: validation and comparison with video observation. Medical and Biological Engineering and Computing 37 (3), 304-308.

Basseville, M., Nikiforov, I. V., 1993. Detection of Abrupt Changes: Theory and Application. Englewood Cliffs, NJ: Prentice-Hall.

Bellman, R., 1961. On the approximation of curves by line segments using dynamic programming. Communications of the Association for Computing Machinery (CACM) 4 (6), 284.

Bouten, C., Koekkoek, K., Verduin, M., Kodde, R., Janssen, J., 1997. A triaxial accelerometer and portable data processing unit for the assessment of daily physical activity. IEEE Transactions on Biomedical Engineering $44(3), 136-47$.

Brailovsky, V. L., Kempner, Y., 1992. Application of piecewise regression to detecting internal structure of signal. Pattern recognition 25 (11), 13611370 .

Cappé, O., Moulines, E., June 2009. On-line expectation-maximization algorithm for latent data models. Journal of the Royal Statistical Society: Series B (Statistical Methodology) 71 (3), 593-613.

Cappozzo, A., Catani, F., Leardini, A., Benedetti, M., Croce, U., 1996. Position and orientation in space of bones during movement: experimental artefacts. Clinical Biomechanics 11, 90-100.

Cappozzo, A., U.D.Croce, Leardini, A., Chiari, L., 2005. Human movement analysis using stereophotogrammetry: Part 1: theoretical background. Gait \& Posture 21, 186-196.

Chamroukhi, F., Samé, A., Govaert, G., Aknin, P., 2009. Time series modeling by a regression approach based on a latent process. Neural Networks $22(5-6), 593-602$.

Chen, K., Xu, L., Chi, H., 1999. Improved learning algorithms for mixture of experts in multiclass classification. Neural Networks 12 (9), 1229-1252. 
Cover, T., Hart, P., 1967. Nearest neighbour pattern classification. IEEE Transactions on Information Theory 13, 21-27.

Dana Kulić, W. T., Nakamura, Y., May 19-23 2008. Combining automated on-line segmentation and incremental clustering for whole body motions. In: In proceedings of the IEEE International Conference on Robotics and Automation. Pasadena, CA, USA, pp. 2591-2598.

Dempster, A. P., Laird, N. M., Rubin, D. B., 1977. Maximum likelihood from incomplete data via the EM algorithm. Journal of The Royal Statistical Society, B 39(1), 1-38.

Eamonn Keogh, Selina Chu, D. H., Pazzani, M., 1993. Segmenting Time Series: A Survey and Novel Approach. Published by World Scientific, Ch. In an Edited Volume, Data mining in Time Series Databases, pp. 1-22.

Enschede, B., 2009. Mti and mtx user manual and technical documentation. URL WWW . Xsens.com

Fod, A., Mataric, M. J., Jenkins, O. C., 2002. Automated derivation of primitives for movement classification. Autonomous Robots 12 (1), 39-54.

Foerster, F., Sep. 1999. Detection of posture and motion by accelerometry: a validation study in ambulatory monitoring. Computers in Human Behavior 15 (5), 571-583.

Fridman, M., 1993. Hidden markov model regression. Tech. rep., Institute of mathematics, University of Minnesota.

Green, P., 1984. Iteratively reweighted least squares for maximum likelihood estimation, and some robust and resistant alternatives. Journal of The Royal Statistical Society, B 46 (2), 149-192.

Hirata, Y., Komatsuda, S., Kosuge, K., September 2008. Fall prevention control of passive intelligent walker based on human model. In: Proceedings of the IEEE/RSJ International Conference on Intelligent Robots and Systems. pp. 1222-1228.

Hussein, S., Granat, M., 2002. Intention detection using a neuro-fuzzy emg classifier. IEEE Engineering in Medicine and Biology Magazine 21 (6), $123-129$. 
Jovanov, E., Milenkovic, A., Otto, C., de Groen, P., Mar. 2005. A wireless body area network of intelligent motion sensors for computer assisted physical rehabilitation. Journal of NeuroEngineering and Rehabilitation 2 (1), $6+$.

Kangas, M., Konttila, A., Lindgren, P., Winblad, I., Jamsa, T., Aug. 2008. Comparison of low-complexity fall detection algorithms for body attached accelerometers. Gait \& Posture 28 (2), 285-291.

Kavanagh, J., Menz, H., 2008. Accelerometry: a technique for quantifying movement patterns during walking. Gait \& Posture 28, 1-15.

Kohlmorgen, J., Lemm, S., 2002. A dynamic hmm for on-line segmentation of sequential data. In: In Advances in Neural Information Processing Systems (NIPS). pp. 793-800.

Kostov, A., Andrews, B., Popovic, D., Stein, R., Armstrong, W., 1995. Machine learning in control of functional electrical stimulation systems for locomotion. IEEE Trans. Biomed. Eng. 42, 541-551.

Kozina, S., Lustrek, M., Gams, M., 2011. Dynamic signal segmentation for activity recognition. In: STAMI (Space, Time and Ambient Intelligence), workshop at 22nd International Joint Conference on artificial Intelligence (IJCAI), Barcelona. pp. 93-98.

Krishnapuram, B., Carin, L., Figueiredo, M., Hartemink, A., 2005. Sparse multinomial logistic regression: fast algorithms and generalization bounds. IEEE Transactions on Pattern Analysis and Machine Intelligence 27 (6), 957-968.

Kulić, D., Nakamura, Y., Sept, 22-26 2008. Scaffolding on-line segmentation of full body human motion patterns. In: In proceedings of the IEEE/RSJ International Conference on Intelligent Robots and Systems. Nice, France.

Lau, H.-y. Y., Tong, K.-y. Y., Zhu, H., Aug. 2009. Support vector machine for classification of walking conditions of persons after stroke with dropped foot. Human movement science 28 (4), 504-514.

Lin, J. F.-S., Kulić, D., september 2011. Automatic human motion segmentation and identification using feature guided hmm for physical rehabilitation exercises. In: Robotics for Neurology and Rehabilitation, Workshop 
at IEEE/RSJ International Conference on Intelligent Robots and Systems (IROS) 2011.

Lin, J. F.-S., Kulić, D., 2011. Automatic human motion segmentation and identification using feature guided hmm for physical rehabilitation exercises. In: In Robotics for Neurology and Rehabilitation, Workshop at the IEEE/RSJ International Conference on Intelligent Robots and Systems. San Francisco, California.

Lindemann, U., Hock, A., Stuber, M., Keck, W., Becker, C., 2005. Evaluation of a fall detector based on accelerometers: A pilot study. Med. Biol. Engineering and Computing 43 (5), 548-551.

Long, X., Yin, B., Aarts, R. M., Sep. 2009. Single-accelerometer-based daily physical activity classification. In: 2009 Annual International Conference of the IEEE Engineering in Medicine and Biology Society. IEEE, pp. 61076110 .

Mannini, A., Sabatini, A., 2010. Machine learning methods for classifying human physical activity from on-body accelerometers. Sensors 10, 11541175 .

Mathie, M. J., Celler, B. G., Lovell, N. H., Coster, A. C., 2004. Classification of basic daily movements using a triaxial accelerometer. Medical \& biological engineering \& computing 42 (5), 679-687.

McGee, V. E., Carleton, W. T., 1970. Piecewise regression. Journal of the American Statistical Association 65, 1109-1124.

McLachlan, G. J., Krishnan, T., 1997. The EM algorithm and extensions. New York: Wiley.

McLachlan, G. J., Peel., D., 2000. Finite mixture models. New York: Wiley.

Mitchell, T. M., 1997. Machine Learning. McGraw-Hill, New York.

Nicolas Dobigeon, J.-Y. T., Scargle, J. D., 2007. Joint segmentation of multivariate astronomical time series : Bayesian sampling with a hierarchical model. IEEE Transactions on Signal Processing 55 (2), 414-423. 
Noury, N., Barralon, P., Virone, G., Boissy, P., Hamel, M., Rumeau, P., 2009. A smart sensor based on rules and its evaluation in daily routines. Engineering in Medicine and Biology Society Procedia Chemistry 1, 32863289 .

Parkka, J., Ermes, M., Korpipaa, P., Mantyjarvi, J., Peltola, J., Korhonen, I., 2006. Activity classification using realistic data from wearable sensors. IEEE Transactions on Information Technology in Biomedicine 10 (1), 119128.

Pogorelc, B., Gams, M., 2012. Home-based health monitoring of the elderly through gait recognition. Journal of Ambient Intelligence and Smart Environments, (to appear).

Preece, S., Goulermas, J., Kenney, L., D.Howard, K.Meijer, R.Crompton, 2009. Activity identification using body-mounted sensors-a review of classification techniques. Physiol. Meas 30 (4).

Rabiner, L. R., 1989. A tutorial on hidden markov models and selected applications in speech recognition. Proceedings of the IEEE 77 (2), 257-286.

Ravi, N., Dandekar, N., Mysore, P., Littman, M., 2005. Activity recognition from accelerometer data. Proceedings of the National Conference on Artificial Intelligence, MIT Press, 1541-1546.

Rumelhart, D. E., McClelland., J. L., 1986. Parallel distributed processing explorations in the microstructure of cognition. cambridge: Bradford books. Computers in Biology and Medicine.

Scanaill, C., Carew, S., Barralon, P., Noury, N., Lyons, D., Lyons, G., Apr. 2006. A Review of Approaches to Mobility Telemonitoring of the Elderly in Their Living Environment. Annals of Biomedical Engineering 34 (4), $547-563$.

Schwarz, G., 1978. Estimating the dimension of a model. Annals of Statistics $6,461-464$.

Spearman, C., 1904. General intelligence, objectively determined and measured. American Journal of psychology 15, 201-293. 
Stone, H., 1961. Approximation of curves by line segments. Mathematics of Computation 15 (73), 40-47.

Trabelsi, D., Mohammed, S., Chamroukhi, F., Oukhellou, L., Amirat, Y., september 2011. Activity recognition using hidden markov models. In: New and Emerging Technologies in Assistive Robotics, Workshop at the IEEE/RSJ International Conference on Intelligent Robots and Systems, San Francisco, California.

Vapnik, V. N., 1999. The Nature of Statistical Learning Theory (Information Science and Statistics). Springer.

Yang, C. C., Hsu, Y. L., 2010. A review of accelerometry-based wearable motion detectors for physical activity monitoring. Sensors 10, 7772-7788.

Yang, J. Y., Wang, J. S., Chen, Y. P., 2008. Using acceleration measurements for activity recognition: An effective learning algorithm for constructing neural classifiers. Pattern Recognition Letters 29 (16), 2213-2220. 